\title{
BIENESTAR PSICOLÓGICO: EL ESTRÉS Y LA CALIDAD DE VIDA EN EL CONTEXTO LABORAL
}

\author{
María Martha Durán \\ Escuela de Ciencias de la Administración \\ Universidad Estatal a Distancia, Costa Rica \\ mduranr@uned.ac.cr
}

\section{RESUMEN}

El entorno cambiante en que nos desenvolvemos actualmente se ha traducido en un incremento de la competencia y de las exigencias para las organizaciones. Esta situación generan altos niveles de presión, que se convierte en parte de la cotidianidad laboral lo que propicia la aparición de enfermedades profesionales: estrés, Síndrome de Burnout, adicción al trabajo, entre otras, en las que el factor emocional o psicológico juega un papel determinante. Aquí se discutirá respecto a la calidad de vida laboral y el estrés laboral, señalando implicaciones personales y organizacionales, así como estrategias de prevención y afrontamiento en ambos niveles.

PALABRAS CLAVE: CAMBIO, CALIDAD DE VIDA LABORAL, ESTRÉS, SALUD MENTAL, ALTERNATIVAS DE ENFRENTAMIENTO.

\section{ABSTRACT}

The changing environment in which we currently live has been translated into increased competition and demands for organizations. This situation affects employees by generating high levels of pressure, which in turn become an everyday part of work. This leads to the emergence of the so-called occupational diseases: stress, burnout, addiction to work and others. Emotional or psychological factors play a decisive role in such diseases.
This article discusses aspects related to the quality of professional life and occupational stress by pointing out personal and organizational implications as well as prevention and coping strategies at both levels.

KEYWORDS: CHANGE, QUALITY OF PROFESSIONAL LIFE, STRESS, MENTAL HEALTH, COPING STRATEGIES

\section{INTRODUCCIÓN}

El dinamismo de un mundo en donde el cambio y la transformación son permanentes y generan altos niveles de incertidumbre y complejidad, lo ambiguo, impreciso y azaroso se abren camino. Las paradojas, las oportunidades y las amenazas interactúan permanentemente, y el paradigma de lo humano y del éxito también cambian, primando el "tener" sobre el "ser", la gratificación inmediata, el dinero como lo único valioso en la vida y el éxito como meta por alcanzar a toda costa y en poco tiempo.

Asumir este cambio como constante, implica también aceptar la dificultad de darse un momento de quietud frente a la velocidad de los sucesos tecnológicos, económicos y culturales que han incidido en la fragmentación entre el mundo personal, familiar y laboral a lo cual, agregan Dolan, García y Diez $(2005$, p. 6) la "desaparición del mundo espiritual y cultural" en todos los ámbitos pues "no hay tiempo" para estos temas. 
En este entorno, en el mercado se desarrolla una competencia feroz, con múltiples alternativas de productos y servicios que satisfacen las necesidades reales y las que el mismo mercado genera. Para las empresas esto significa el enfrentar nuevos retos de calidad en procesos y productos o servicios, de eficiencia en la gestión y reducción de costos, de innovación y de productividad en general.

La presión organizacional consecuente, se traslada a los trabajadores, potenciado la aparición y desarrollo de una pandemia del mundo actual: el estrés laboral, que impacta en su bienestar psicológico y calidad de vida.

De esta forma dado que la competitividad, las condiciones y ambiente de trabajo y la calidad de vida de trabajador marchan paralelamente, es difícil mantener una empresa en el mercado si los procesos de trabajo deterioran la salud de los trabajadores y genera conflictos.

Si entendemos la Salud como un proceso integral social, físico y mental que se da a lo largo de la vida, asumimos que la forma en que se da el proceso depende en parte de la persona y por otra de las condiciones y oportunidades concretas que encuentre en el medio sociocultural de existencia que facilitan o dificultan esta condición. El resultado se traduce en bienestar psicológico y calidad de vida o en enfermedades físicas y psíquicas.

En el ámbito laboral, el estrés es uno de los responsables directos de las más comunes y mortales enfermedades que afectan al trabajador y la trabajadora del nuevo siglo.

Este es el punto de partida de este documento: la productividad, la eficiencia y la salud laboral reflejadas en el bienestar psicológico y la calidad de vida laboral, se entrelazan e impactan la competitividad de una empresa u organización.
De ahí, la importancia del estrés laboral, su atención y administración tanto en el ámbito personal como organizacional.

\section{La Calidad de Vida Laboral (CVL)}

Desde la perspectiva laboral podemos referirnos a la Calidad de Vida Laboral desde dos posiciones:

1. Como un concepto y una filosofía de organización del Trabajo cuyo fin es mejorar la vida del empleado en las organizaciones más allá del dinero.

Desde este enfoque Andrés Rodríguez (1998) define la Calidad de Vida Laboral como:

(...) un conjunto de estrategias de cambio con objeto de optimizar las organizaciones, los métodos de gerencia y/o los puestos de trabajo, mediante la mejora de habilidades y aptitudes de los trabajadores, fomentando trabajos más estimulantes y satisfactorios y traspasando poder, responsabilidad y autonomía a los niveles inferiores (p. 268).

También se incluyen una compensación razonable y participación en los beneficios de las empresas; la seguridad del empleo; la seguridad e higiene en el trabajo; el reconocimiento del progreso profesional; acuerdos de tiempo flexible; el uso de formas no burocráticas de organización del trabajo, comunicación abierta y retroalimentación, entre otros.

2. Para el trabajador/a es la combinación de necesidades y percepciones acerca de su empresa y las condiciones de ambiente cotidiano y de trabajo: La percepción favorable de apoyo y promoción de su satisfacción, por medio de los sistemas de recompensas, seguridad laboral y oportunidades de crecimiento, y el sentirse respaldado y cuidado por la organización, contar con las condiciones óptimas que faciliten la realización 
de las labores, la necesidad de satisfacer los deseos y expectativas personales, además de la necesidad de mantener buenas relaciones personales

Estos aspectos tienen repercusiones significativas en la calidad de vida y en el equilibrio socioemocional y afectivo de los miembros de la empresa, es decir, en su bienestar psicológico, e impactan en la productividad y la perpetuación de la compañía.

Integrando ambos enfoques en la figura 1, se asumen el compromiso, la confianza y el control como aspectos clave en la organización para el desarrollo de la Calidad de Vida Laboral, y se constituyen en los factores que integran los cuatro ejes de abordaje propuestos: bienestar general, ambiente, aspectos psicosociales y aspectos sociopolíticos.
Según Segurado y Agulló (2002, p. 831) el objetivo primordial de la CVL estriba en "alcanzar una mayor humanización del trabajo a través del diseño de puestos de trabajo más ergonómicos, unas condiciones de trabajo más seguras y saludables, y unas organizaciones eficaces, más democráticas y participativas capaces de satisfacer las necesidades y demandas de sus miembros además de ofrecerles oportunidades de desarrollo profesional y personal", aspectos que generan compromiso y satisfacción con el trabajo, con una sensación de propiedad, autocontrol, responsabilidad y dignidad del trabajador, que propicia una mejor condición de vida en general.

La CVL se traduce en términos operativos mediante programas tanto específicos, de alcance limitado, como de carácter más amplio dentro

\section{FIGURA 1 \\ DIMENSIONES DE LA CALIDAD DE VIDA LABORAL}

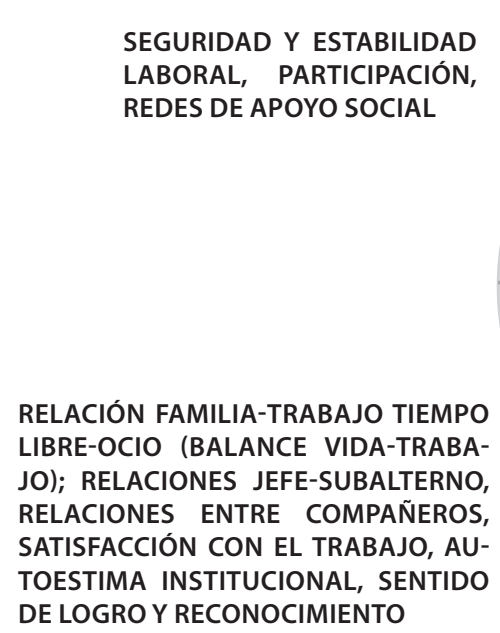

SEGURIDAD Y ESTABILIDAD LABORAL, PARTICIPACIÓN, REDES DE APOYO SOCIAL

RELACIÓN FAMILIA-TRABAJO TIEMPO LIBRE-OCIO (BALANCE VIDA-TRABAJO); RELACIONES JEFE-SUBALTERNO, RELACIONES ENTRE COMPAÑEROS, TOESTIMA INSTITUCIONAL, SENTIDO DE LOGRO Y RECONOCIMIENTO

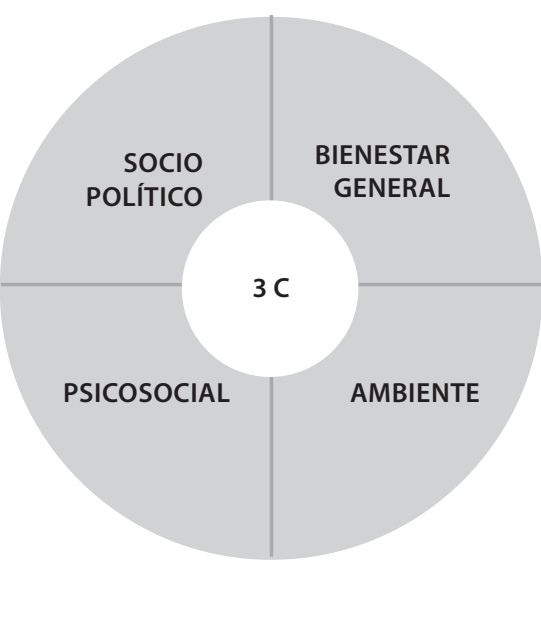

RETRIBUCIÓN, INCENTIVOS Y BENEFICIOS; CRECIMIENTO Y DESARROLLO PROFESIONAL, AUTORREALIZACIÓN, OPORTUNIDAD DE PARTICIPACIÓN CREATIVA, RESPETO Y DIGNIDAD

CLIMA ORGANIZACIONAL: CONDICIONES AMBIENTALES SEGURAS Y CÓMODAS; CONDICIONES ERGONÓMICAS Y SOCIALES; OPORTUNIDAD QUE TIENE EL INDIVIDUO PARA USAR Y DESARROLLAR SUS CAPACIDADES

3 C: COMPROMISO, CONFIANZA Y CONTROL

FUENTE: Elaboración propia 
de la organización que pueden incluir: seguridad e higiene en el trabajo, procedimientos y sistemas de seguridad, entrenamiento y capacitación para el desempeño de la profesión u oficio, instalaciones adecuadas y agradables, desarrollo social y personal, remuneración justa, integración familiar, recreación y deporte, ergonomía, tecnología adaptable, comunicación individuoempresa, estabilidad laboral, el balance adecuado de trabajo y vida personal, entre otros.

\section{El Estrés}

Entendemos por estrés una respuesta adaptativa, mediada por las características individuales y/o procesos psicológicos, la cual es a la vez consecuencia de alguna acción, de una situación o un evento externo que plantean a la persona especiales demandas físicas y/o psicológicas. Se asume así como un "mecanismo de defensa" que conlleva un conjunto de reacciones de adaptación del organismo.

Korman propuso en 1974 (citado por Ivancevich y Matteson, 1992) que existe un nivel de estrés óptimo para cada persona (Euestres), de manera que si esta mantiene un nivel de activación por encima o por debajo de ese nivel repercutirá negativamente sobre el desempeño laboral (relación de " $U$ " invertida entre activación y desempeño que establece que "la productividad mejora al incrementarse la tensión, hasta cierto punto en que se deteriora conforme el estrés se aleja de lo óptimo"). Por lo tanto muy poca o demasiada tensión (Diestres) pueden resultar en una disminución de la productividad.

La tensión surge también cuando se da un alejamiento de las condiciones de estrés óptimas, que no puede ser fácilmente corregido y causa un desequilibrio entre demanda y capacidad de control. Para que se den casos serios de tensión, la persona debe considerar como serias las consecuencias de no poder satisfacer las demandas.
Autores como Dolan, García y Díez (2005) y Bresó (2008) incorporan algunos factores emocionales, conductuales y cognitivos que pueden actuar como "amortiguadores" del estrés de una persona:

- Autoestima, Autonomía, percepción de Autoeficacia

- Apoyo social: emocional, material o físico

- Humildad y humor, como parte de una buena autoestima

Así, integrando estos diferentes abordajes y propuestas (Ivancevich y Matteson, 1992; Rodríguez, 1999; Dolan et al. 2005; Bresó, 2008), podemos representar el grado de estrés de una persona de la siguiente forma ${ }^{1}$.

\section{$E=D-(3 A=S 2 H)$}

En donde

E: estrés

D: demandas o compromisos adaptativos (estresores)

R: recursos de control y afrontamiento situacional

A: autoestima, autonomía y percepción de autoeficacia

S: calidad del apoyo social percibido

$\mathrm{H}$ : humildad y humor

De esta forma se asume que el estrés tiene diferentes grados o manifestaciones, y que estas están en relación con las demandas o compromisos adaptativos (estresores potenciales) y los recursos de control y afrontamiento situacional con que cuenta, que son afectados por factores personales.

1. Esta fórmula de estrés es con fines nemotécnicos, NO es una fórmula matemática. 


\section{Ciclo del estrés}

Una experiencia se considera como estresante cuando se percibe cognoscitivamente como retadora, desafiante, amenazante, dañina o desagradable para la persona. Las personas se diferencian por sus reacciones al estrés, dependiendo del significado psicológico que tenga el evento para ellos. El grado de ansiedad que experimenta el individuo se determina por la relevancia de la situación. Un ejemplo son las situaciones de logro en las cuales el estrés puede depender de las consecuencias del éxito o fracaso.

El estrés se puede entender en términos de cuan importante es el estresor para la persona y hasta donde el evento estresor puede controlarse, es decir, qué habilidad tiene la persona para responder al estrés (ver Figura 2).
La respuesta al estrés es el esfuerzo de nuestro cuerpo por adaptarse al cambio o, con mayor precisión, por mantener la homeostasis. Experimentamos estrés cuando no podemos mantener o recuperar este equilibrio personal.

En condiciones de vida como las que actualmente tenemos no es posible eliminar el estrés; lo que si podemos hacer es desarrollar habilidades y estrategias tanto individualmente como en la organización, para manejar y controlar de una manera más sana las situaciones de estrés negativo, es decir, crear defensas mediante el conocimiento de las reacciones del organismo ante estas situaciones y aprender a manejarlo utilizando diferentes técnicas, para una mayor productividad y mejores resultados de un estado óptimo.

FIGURA 2

\section{CICLO DEL ESTRÉS}

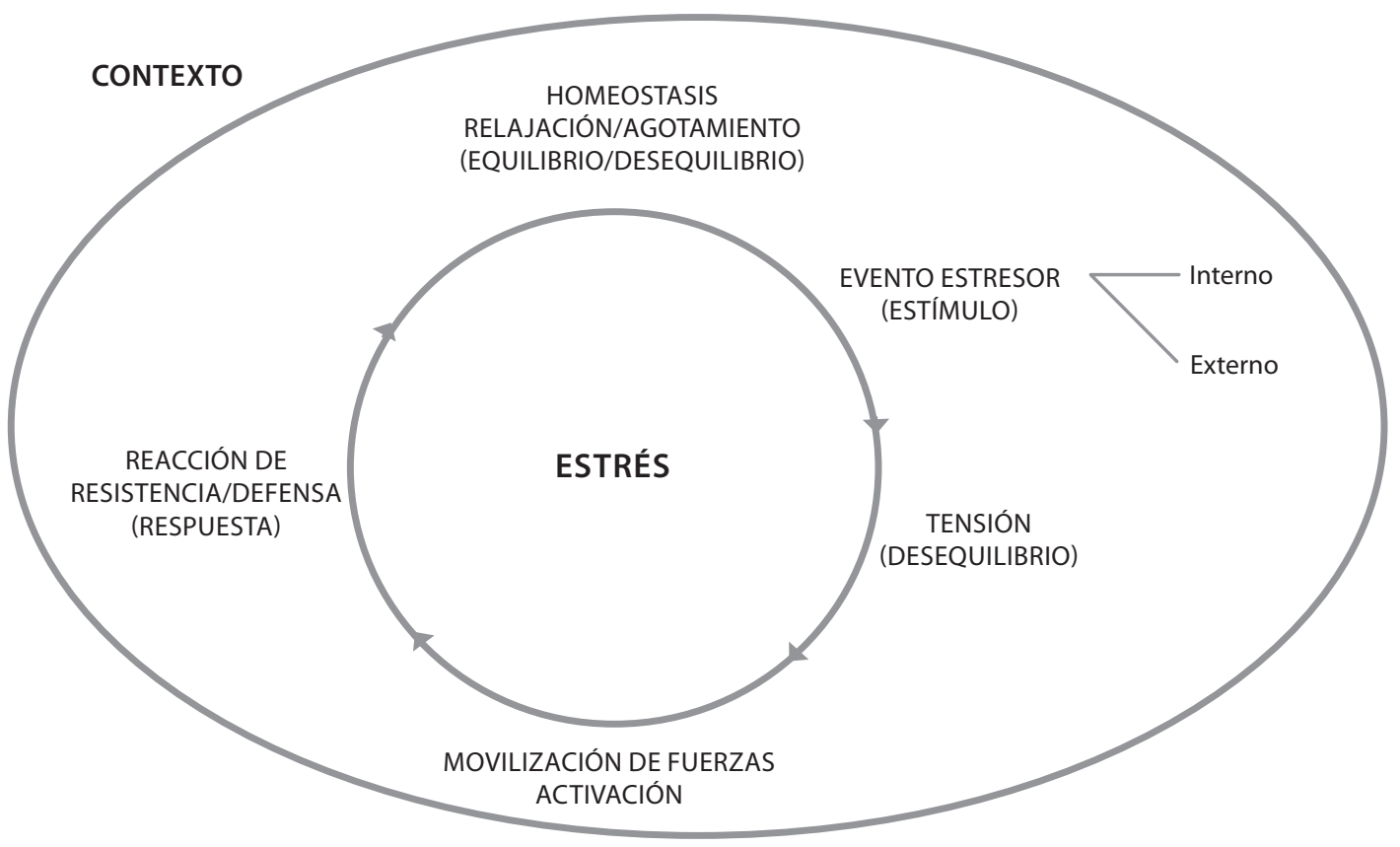

FUENTE: Elaboración propia 
El estrés en el trabajo genera en las empresas una serie de consecuencias negativas en su productividad al igual que en la motivación, satisfacción y compromiso laboral de sus colaboradores. Se asocia con un incremento de accidentes, ausentismo, incapacidades y enfermedades ocupacionales. Propicia más conflictos interpersonales en el trabajo y en la familia, mayor índice de errores, incide en la toma de decisiones, en el bajo rendimiento ocupacional, distanciamiento afectivo con clientes, aumento en los gastos en materia de salud y rotación de personal, entre otros aspectos.

Desde esta perspectiva, autoadministrar el estrés significa, en última instancia, tener control sobre la propia vida, manejando efectivamente tanto los síntomas como las causas del estrés en los diferentes ámbitos de la vida. El "barómetro" que permitirá diferenciar el "euestres" del "diestres" es el bienestar: el estado físico, psíquico, moral de una persona en concreto.

\section{Estrés Laboral}

Ampliando el concepto de Estrés para ubicarlo en un contexto específico, entendemos por Estrés Laboral un desequilibrio percibido entre las demandas laborales y la capacidad de control, aspiraciones de la persona y la realidad de sus condiciones de trabajo que generan y una reacción individual congruente con la percepción del estresor (es) laboral (es).

Es, entonces, una respuesta adaptativa: una reacción individual del trabajador/a ante una situación relacionada con su trabajo, percibida y valorada como un desafío o reto positivo, o como amenazante o dañina, a partir de la relación entre las demandas laborales y los recursos necesarios para atenderla. La percepción de fal- ta de control sobre la situación y el sentimiento de sobreesfuerzo, originan desgaste por exceso de activación, manifestándose de diferentes formas (físicas, emocionales y conductuales). Las percepción y valoración esta mediada por las características personales (diferencias individuales) frente a las exigencias de las tareas en sus diferentes grados, así como la percepción de logro resultante.

En situaciones laborales todos tenemos diferentes grados de estrés. Lo que debe propiciar la persona y la organización es la preponderancia de condiciones que impulsen un estrés positivo (euestrés).

Basados en Bresó (2008) y Dolan et al. (2005) podemos indicar tres factores intervinientes en el Estrés Laboral (ver Figura 3):

1. Recursos personales: refiere a las características del trabajador tales como habilidades, aspiraciones, necesidades, valores, creencias de eficacia personal (autoeficacia), capacidad de control del medio (locus de control), entre otros aspectos.

2. Recursos laborales: son las características del trabajo que reducen las demandas y sus costos asociados; $y$ / o estimulan el crecimiento personal, el aprendizaje y la consecución de metas

3. Demandas laborales: entendidas como las características del trabajo que requieren un esfuerzo físico y/o psicológico (mental y emocional) y por tanto tiene costes físicos y/o psicológicos 
FIgURA 3

\section{FACTORES INTERVIENEN EN EL ESTRÉS LABORAL}

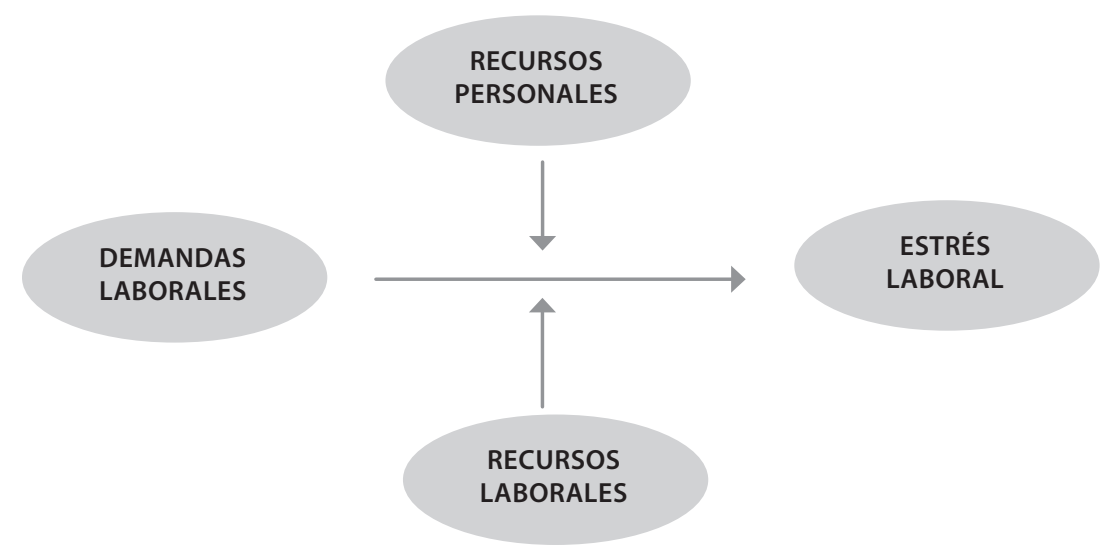

FuENTE: Adaptado de Bresó (2008)

El balance o desbalance de estos factores incidirá en el tipo y grado de estrés que una persona viva.

\section{Estresores}

Por estresor se entiende el agente interno o externo (estímulo) que, producto de factores cognitivos y perceptuales, la persona evalúa y conceptualiza en términos de las relaciones propias con los eventos, y provoca una respuesta de estrés. En este sentido, todo suceso, situación o persona que encontramos en nuestro medio externo puede ser un estresor; aunque abundan los estresores potenciales, los estresores reales son mucho menos numerosos.

Hay muchos modos de categorizar las condiciones de vida potencialmente estresantes. Para facilitar la identificación de los estresores asociados, se han definido tres niveles de análisis (Ivancevich \& Matteson, 1992; Dolan et al. 2005):
1. Estresores individuales: considera los aspectos personales (a partir de las diferencias individuales) que influyen en la interpretación subjetiva tanto de lo que se considera como estrés y de la reacción en consecuencia:

a) Cognitivo/afectivas: por ejemplo creencias y valores propios, aspiraciones, tipo de personalidad, locus de control, tolerancia a la ambigüedad, niveles de necesidad, autoestima.

b) Conductuales: habilidades, tolerancia a la ambigüedad, asertividad entre otros.

c) Demográficas: edad, género, educación, ocupación, estado de salud, total de horas trabajadas, entre otros.

2. Estresores del entorno o extraorganizativos: comprende factores políticos, económicos, sociales y familiares que inciden sobre la persona (problemas como las guerras, el terrorismo, el hambre, la pobreza, el deterioro del medio ambiente, el cambio climático, los problemas en la educación, la salud, la 
exclusión, las crisis financieras y sociales, la corrupción, la violación de los derechos humanos en general, entre otros).

3. Estresores organizativos: pueden ser agrupados en cuatro tipos:

a) Estresores del ambiente físico como luz, ruido excesivo, vibraciones, humedad, disponibilidad y disposición del espacio físico, entre otros.

b) Estresores de nivel individual: tipo de trabajo², sobrecarga de trabajo, conflictos y ambigüedad de roles, responsabilidad por gente, discrepancias con las metas de la carrera laboral, manejo de idiomas.

c) Estresores de nivel grupal (interpersonales): en cuanto al trabajo de grupo: conflicto grupal, presiones del grupo, posibilidades de interacción con los colegas (en el trabajo, en los descansos y después del trabajo); tamaño y cohesión del grupo primario de trabajo; reconocimiento por el trabajo realizado; apoyo social; apoyo instrumental; carga de trabajo equitativa; acoso.

d) En cuanto a supervisión: participación en la toma de decisiones; recibir retroalimentación y reconocimiento del supervisor; ofrecer retroalimentación al supervisor; cercanía de la supervisión; exigencias poco claras o contradictorias; acoso.

2. Por ejemplo los trabajos que implican contacto con padecimientos, enfermedades o lesiones de las personas (por ejemplo la policía o los servicios médicos de urgencia) o en los que el propio personal hace frente a un peligro físico (buzos, trabajadores de la industria pesquera) o a amenazas de violencia (guardas, jurados) pueden constituir pesadas cargas emocionales para los empleados y producir estrés. e) Estresores organizativos entre los que destacan las siguientes dimensiones:

- Condiciones relativas a la empresa tales como estructura; puesto directivo (frente a puesto en la cadena de producción); prestigio relativo del puesto; estructura empresarial poco clara; burocracia organizativa; políticas discriminatorias, estilos gerenciales, tecnología.

- Aspectos temporales de la jornada laboral y del propio trabajo: trabajo nocturno y por turnos; horas extraordinarias no deseadas o "excesivas"; el pluriempleo; un ritmo rápido de trabajo, especialmente cuando hay que estar muy vigilante; tiempos de entrega; programación de los ciclos de trabajo y de reposo; la variación de la carga de trabajo;

- Contenido del trabajo: trabajo fragmentado, repetitivo y monótono, con poca variedad de las tareas o de la aplicación de conocimientos; tareas o exigencias poco claras o contradictorias; recursos insuficientes para las exigencias o responsabilidades del trabajo.

- Otros aspectos: relación entre incentivos y rendimiento (evaluación del desempeño), controles de calidad, traslados de lugar de trabajo dentro y fuera del país, estabilidad laboral, diseño del puesto de trabajo, entre otros.

Es importante señalar que tanto el exceso como la carencia asociada con la activación del trabajador pueden ser considerados como estresantes. De esta forma la mucha o poca dificultad en el trabajo, la mucha o poca responsabilidad o participación en la toma de decisiones, pueden ser fuente de estrés en el trabajo. 


\section{Consecuencias del estrés laboral}

Los costos del estrés laboral pueden ser muy altos tanto desde su valor personal como empresarial u organizacional. Se puntualizan aquí algunos de los aspectos mencionados en apartados anteriores, sin agotar las posibilidades de manifestaciones:

1. En el ámbito personal:

- Salud física: trastornos gastrointestinales, cardiovasculares, respiratorios, endocrinos, dermatológicos, musculares, aumento del ritmo cardíaco y presión sanguínea, sudoración, sequedad de la boca, escalofrío, dilatación de pupilas, tensión muscular, insomnio o hipersomnia, alergias, úlceras, etc.

- Salud Mental (emocional/cognitivo): frustración, ansiedad/angustia, depresión, enojo/irritabilidad, baja autoestima, culpa, incapacidad para tomar decisiones y concentrarse, olvidos frecuentes, hipersensibilidad, bloqueo mental.

- Aspectos Conductuales: irritabilidad y mal humor, adicciones, agresividad, apatía.

- Dimensión Social: distanciamiento y/o dificultades en las relaciones de pareja, familia, amigos y compañeros de trabajo.

2. En el ámbito organizativo: disminución de producción, ya sea en cantidad, calidad o ambas; falta de cooperación entre compañeros, aumento de peticiones de cambio de puesto de trabajo; necesidad de una mayor supervisión del personal; aumento de quejas y de conflictos, incremento de costos en salud (incapacidades, médico de empresa y otros), aumento de ausentismo, accidentes e incidentes.

Propicia más conflictos interpersonales, mayor índice de errores, bajo rendimiento ocupacional, distanciamiento afectivo con clientes, aumento en los gastos en materia de salud y rotación de personal, entre otros aspectos $^{3}$.

Se incrementan el acoso moral y la violencia en el trabajo (mobbing o acoso psicológico); también es frecuente que se presenten casos de Síndrome del Burnout (Quemado o agotamiento emocional producido por agotamiento laboral y actitudes negativas hacia el desempeño laboral), lo cual remite también a diferentes grados de fatiga fisiológica y patológica que pueden ocasionar envejecimiento prematuro, con reducción de esperanza de vida, accidentes de trabajo y muerte ${ }^{4}$, además de enfermedades profesionales y adicciones (alcohol, tabaco, trabajo, sexo, comida, gasto, internet, juego...)

Todas estas condiciones han incidido en que la Organización Mundial de Salud (2005) destacara que el estrés en el trabajo se constituye en una de las máximas prioridades en el ámbito mundial en el campo laboral pues se espera para el futuro un incremento progresivo del mismo por las condiciones actuales del mercado y los retos que impone el mundo de los negocios del nuevo siglo, constituyéndose en un peligro para las economías de los países industrializados y en vías de desarrollo en tanto que los efectos del estrés lesionan la productividad y competitividad de las empresas, al afectar la salud física y mental de los trabajadores, lo cual les ocasiona

3. Dolan et al. (2005, p. 74) señalan que "el estrés profesional resulta del exceso de compromisos laborales y personales (externa e internamente condicionados) asociado a la falta de recursos psicológicos de control o afrontamiento situacional, ya al descuido de la construcción de un buen apoyo social a lo largo del tiempo".

4. Por la disminución de la posibilidad de enfrentar situaciones inesperadas (Comunicación personal con Arce, 2004) 
costos económicos y humanos muy altos a las organizaciones (Del Pino, 2005). Un lamentable ejemplo es la situación de los 24 suicidios en la empresa France Telecom, en el período febrero 2008 a 28 de setiembre 2009, atribuidos a los métodos de trabajo de esta transnacional y a su política de reorganización de recursos humanos.

\section{Enfrentando el estrés}

\section{La administración personal}

Partiendo de la definición de estrés laboral expuesta, se asume que es necesario abordar aspectos tanto preventivos como paliativos en su manejo, considerando los niveles personal, social y organizacional en un momento y lugar determinados. El aprendizaje es un elemento integrador en este proceso, ya que se requiere de aprender, desaprender y reaprender tanto aspectos cognitivos, conductuales y emocionales: autoconocimiento, control emocional, asertividad, autoestima y creatividad por ejemplo.

Desde esta perspectiva, se abordan las estrategias personales de acción antes y durante situaciones de estrés desde el Modelo sobre el Ciclo del Estrés que se propuso en páginas anteriores. Es así como la gestión preventiva individual del estrés puede ir dirigida a:

a) El evento estresor para lo cual debe identificarse si son externos, internos o ambos interactuando pues el abordaje es diferente. Por ejemplo el disminuir o cambiar la entrada de estresores externos implica cambio ambiental, mientras que al aumentar los recursos disponibles de la persona para lidiar con los estresores el manejo es desde el nivel intrapersonal.

b) La movilización de fuerzas, en cuanto a formas personales y particulares de activación: activación de las "señales de alerta" y niveles de tolerancia (umbral al estrés). c) La respuesta, refiriéndose a los tipos de reacciones de la persona, según el estresor o la situación, así como la identificación de estrategias cognitivas, emocionales y comportamentales para modificar esas respuestas y estilos de vida patógenos asociados. Esto puede requerir de apoyo profesional (por ejemplo técnicas de resolución de problemas, asertividad, reestructuración cognitiva, gestión del tiempo, técnicas de relajación, entre otros.)

d) La homeostasis: desarrollo y mantenimiento de una buena salud y hábitos: mantenerse activo física y mentalmente, dieta adecua$\mathrm{da}$, aprender a relajarse, dormir bien, apoyo social. La planificación de tiempo y dinero (ingresos y egresos-inversiones y gastos) es parte de este equilibrio. El redefinir metas personales según la realización personal y profesional, presupuesto, familia, vida social y recreativa, buscando el balance vida personal, familia, trabajo, ocio y recreación. Además es necesario poder identificar nuestro propio "barómetro" de cuándo y cómo nos afecta el estrés; la opinión profesional puede ayudar a calibrar este barómetro personal pero es cada persona la que debe monitorearlo.

e) El contexto: implica la consideración del tiempo (duración de la situación de estrés), el lugar, la persona(s) y el momento o situación específica.

\section{El papel de la organización}

Es evidente que la mayoría de los factores de riesgo en el ámbito profesional proceden del entorno laboral, de la forma en que la organización estructura sus tareas y responsabilidades, y del modo en que se organizan los diferentes procesos desarrollados en ella. Por esto es necesario intervenir directamente en el nivel organizacional como forma de prevenir y controlar la 
salud laboral y los problemas de salud mental en general.

El control y la prevención en salud laboral (y especialmente en salud mental en general y estrés en específico), requieren de un cambio en la filosofía de la organización dirigido a incrementar la calidad de vida laboral (CVL) y a potenciar a su personal como condición básica para optimizar y rentabilizar los diferentes recursos involucrados. El personal podría canalizar adecuadamente su energía y potencial productivo ante las demandas adversas y exigentes del ambiente laboral actual.

Lo anterior visibiliza la necesidad actual de que las organizaciones lleven a cabo el establecimiento de programas y políticas sobre salud laboral encaminadas a la creación, difusión y establecimiento de una cultura organizacional, en la cual se tome en cuenta en forma adecuada aspectos de salud ocupacional, seguridad e higiene industrial y de protección ambiental; acciones enfocadas a contribuir al mejoramiento de las condiciones laborales, las cuales permitirían disminuir los altos costos económicos en esta materia que afectan las utilidades de las empresas, así como lograr y mantener un estado de bienestar psicológico y físico del personal en su ambiente laboral para el beneficio organizacional.

Es por esto que el control y la prevención, intervención e investigación en Salud Laboral y especialmente del estrés, requieren de un cambio en la filosofía de la organización dirigido a incrementar la calidad de vida laboral y a potenciar a su personal como condición básica para optimizar y rentabilizar los diferentes recursos involucrados. Esto implica la claridad de obtener no solo altos niveles de productividad, sino también el involucramiento, la satisfacción y motivación de los trabajadores mediante el manejo de la Cultura Organizacional.
La Organización Mundial de la Salud (Leka rt al. 2004) señala que el Estrés Laboral puede reducirse en la organización, considerando tres niveles de prevención:

- Prevención primaria: implica la atención e intervención en aspectos ergonómicos, la descripción de puestos de trabajo y diseño ambiental, y el mejoramiento de la organización y la gestión.

- Prevención secundaria: se focaliza en la formación y capacitación de los trabajadores tanto a nivel individual como organizacional. Las estrategias individuales se orientan a la adquisición de estrategias instrumentales para la solución de problemas (asertividad, manejo del tiempo, entre otros), además de programas dirigidos a estrategias de carácter paliativo enfocadas en la adquisición de destrezas para el manejo de emociones (entrenamiento en relajación, expresión de la ira, manejo de sentimiento de culpa, y otros).

- Prevención terciaria: relacionada con la reducción del estrés laboral mediante el desarrollo de sistemas de gestión más sensibles y con mayor capacidad de respuesta, además de la mejora en la prestación de servicios de salud ocupacional.

Sin agotar las posibilidades, algunos de los temas a considerar son:

- Mejorar la selección, inducción y promoción del personal.

- Mejorar la descripción de puestos y claridad en los roles profesionales.

- Establecer programas de formación y desarrollo.

- Desarrollar programas de enriquecimiento del trabajo y planes de carrera más innovadores. 
- Descentralizar la toma de decisiones, aumentando la participación y mejorando la supervisión.

- Potenciar el desarrollo de los trabajadores, manteniendo y ampliando sus competencias, aumentando la variedad de habilidades y autonomía.

- Mejorar los sistemas de evaluación del desempeño; los programas de incentivos y beneficios y de motivación y satisfacción laboral.

- Desarrollar sistemas de retribución más equitativos.

- Ofrecer horarios de trabajo flexibles y considerar el teletrabajo.

- Establecer programas de bienestar que incluyan la disminución del estrés en el lugar de trabajo mediante prácticas sanas de gestión; idear políticas de gestión del personal que tengan el propósito directo de mejorar la salud mental en el trabajo; realizar sesiones de información para las juntas directivas de las empresas, en las que se exponga el impacto de la salud mental en el desempeño de la empresa.

A pesar de su importancia, la gestión organizacional del estrés enfrenta importantes obstáculos:

1. la poca conciencia sobre su importancia tanto para los trabajadores como para la organización, asociado a la importancia (y carencia) de un buen diagnóstico;

2. la rentabilidad económica pues en general no es prioritaria la atención a la Salud Laboral en tanto no se identifica la relación con la productividad y la eficiencia de los trabajadores;
3. también se presentan casos en que las propuestas de cambio poco planificadas, sin adaptarse a los esquemas de utilidad, rentabilidad y practicidad necesarios en toda empresa, es decir, sin propiciar la convergencia entre las metas de la organización y las de la prevención en la empresa.

\section{CONCLUSIONES}

¿Es posible administrar el estrés y mejorar la calidad de vida en el contexto laboral actual?

Sí, es posible. Aunque en un entorno cada vez más complejo las estrategias deben ser más claras y constantes y atender los dos niveles de abordaje, el individual y el organizacional. Así se evitará sucumbir en uno de los problemas de salud mental más importantes del siglo XXI.

El estrés es inevitable en tanto es un esfuerzo de adaptación ante diferentes estímulos que se asumen como estresores. Desde esta perspectiva, tanto los cambios percibidos como positivos o como negativos, producen estrés.

Lo que sí es posible evitar es la situación de estrés prolongado, recurrente y su transformación en estrés intenso y crónico. En definitiva, lo que hay que preguntarse es: ¿Qué intensidad tiene la situación estresante? ¿Cuánto dura? ¿Es adecuado a las exigencias de la situación? ¿Desde el punto de vista de quién? ¿Cuáles son los principales estresores? ¿Qué se puede hacer para minimizar su impacto?

Si bien es cierto que los trabajadores se sienten más satisfechos cuando su entorno laboral es seguro y confortable (Dolan et al. 2005), lo cual tiene un impacto directo en el incremento de la calidad en el trabajo, también lo es que dentro de los factores y condiciones psicosociales en el contexto de vida actual se dan múltiples y varia- 
das fuentes de estrés que pueden resultar nocivas para la salud y el bienestar de las personas.

El estrés laboral que produce resultados negativos afecta el rendimiento en el trabajo y produce elevados costos (visibles y ocultos) para la empresa en momentos en que socialmente hay mayor conciencia de la corresponsabilidad de las organizaciones del estrés de sus empleados, y de las consecuencias que pueda tener para ellos.

Las estrategias de atención van dirigidas a la disminución de los efectos negativos derivados de la percepción del estrés, para lo que se utilizan técnicas dirigidas a modificar la valoración subjetiva del trabajador de los estímulos del entorno y de los recursos disponibles para hacerles frente, orientándose hacia la búsqueda, selección, aprendizaje y emisión de respuestas adaptativas. De esta forma los programas son individuales pues la eficacia de los mismos depende de la puesta en práctica por parte del trabajador. Pero también la definición de programas de Calidad de Vida Laboral y políticas congruentes.

Sin embargo, el desarrollo de estrategias de atención al estrés laboral requiere de un paso fundamental: que las organizaciones reconozcan y acepten que la Salud Mental es un tema importante, asumiendo desde la alta gerencia el compromiso de promoverla, y que se concrete en la realización de políticas que permitan mayor y mejor comunicación, autonomía, participación, flexibilidad laboral y sistemas de promoción e incentivos, incidiendo así en la productividad y eficiencia, aumentando la competitividad.

\section{REFERENCIAS}

Arce, E. (octubre, 2004) Comunicación personal. Universidad de Costa Rica.

Ballesteros, B; Mediana, Argelia; Caycedo Claudia (2006). El bienestar psicológico definido por asistentes a un Servicio de Consulta Psicológica en Bogotá, Colombia. Universitas Psychologica, vol.5 no.2 Bogotá Jan./May 2006. Recuperado el 23 de octubre de 2009 de http://www.scielo. org.co/scielo.php?pid=\$1657-92672006000200 004\&script=sci_arttext

Bresó, E. (2008). Taller: "Del Burnout al Engagement". Sistema de Estudios de Posgrado. Universidad de Costa Rica.

Buendia, J. \& Ramos, F. (2001). Empleo, estrés y salud. Madrid: Editorial Pirámide.

Cifre, E. (1999). Bienestar psicológico, características del trabajo y nuevas tecnologías: validaciónampliación del modelo vitamínico de Warr. Tesis doctoral. Castellón: Publicaciones de la Universitat Jaume I. Recuperado el 16 de setiembre de 2009 de http://www.tesisenxarxa.net/ TDX/TDX_UJITESIS/AVAILABLE/TDX-0527108094150/cifre.pdf

Del Pino, R. (2005). Administración estratégica del estrés en el trabajo: diagnóstico de estresores laborales y apoyos institucionales en complejos petroleros marinos de producción. Ponencia para el Congreso Internacional de Contaduría, Administración e Informática. Universidad Nacional Autónoma de México.

Dolan, S., García, S. \& Díez, M. (2005). Autoestima, estrés y trabajo. España: Mac Graw Hill.

Dolan, S., Martín, I. \& Soto, E. (2004). Los 10 mandamientos para la Dirección de Personas. España: Editorial Gestión 2000.

Dolan, S., Valle, R., Jackson, S. \& Schuler, R. (2003). La Gestión de los Recursos Humanos: preparando profesionales para el Siglo XXI. España: Mac Graw Hill. 
González, J. M, \& Rodríguez, A. (s.f.) Los cambios en el entorno y sus repercusiones sobre el mercado laboral. Recuperado el 01-03-2008 de http://www. uhu.es/aeurla/revista/art101.doc.

Guillén, C.(2000). Psicología del Trabajo para las relaciones laborales. 2da. Edición. España: Editorial McGraw-Hill.

Ivancevich, J. \& Matteson, M. (1992). Estrés y trabajo: una perspectiva gerencial. México: Editorial Trillas, S.A.

Leka, S; Griffiths, A; Cox, T (2004). La organización del trabajo y el estrés. Francia: Organización Mundial de la Salud. Recuperado el 10-03-2006 de http:// www.who.int/occupational_health/publications/pwh3sp.pdf

Madl, H., Winkler, K. \& Schnurer, K. (2004) Einleitung ins individuelle Wissensmanagement-Instrumente. Alemania: InWEnt-Capacity Building Internacional.

Peiró, J. \& Prieto, F. (1996). Tratado de Psicología del Trabajo Vol. 1. La Actividad Laboral en su Contexto. Madrid, España: Editorial Síntesis S. A.

Peiró, J. \& Prieto, F. (1998). Tratado de Psicología del Trabajo Vol. 1. La Actividad Laboral en su Contexto. Vol II: Aspectos Psicosociales del Trabajo. Editorial Síntesis Psicología. Primera edición.
Rampersad, H. (2004). Cuadro de mando integral, personal y corporativo (Total Performance Scorecard, TPS). España: Mac Graw Hill.

Robbins, S. (2004). Comportamiento Organizacional. México: Prentice Hall.

Rodríguez, A. (1999). Introducción a la Psicología del Trabajo y de las Organizaciones. España: Ediciones Pirámide.

Salanova, M; Martínez, I; Bresó, E; Llorens, S \& Grau, R. (2005). Bienestar psicológico en estudiantes universitarios: facilitadores y obstaculizadores del desempeño académico. Revista Anales de Psicología, 2005, vol. 21, no 1 (junio), 170-180. Recuperado el 01-03-2008 de http://www.um.es/ analesps/v21/v21_1/16-21_1.pdf

Segurado A. Agull, E. (2002). Calidad de vida laboral: hacia un enfoque integrador desde la Psicología Social. Psicothema, 14, (4), pp. 828-836.

Wilkerson, B. (2000). La salud mental como arma fundamental de la productividad. En "Día Mundial de la Salud Mental: 10 de octubre de 2000. Federación Mundial para la Salud Mental."Taiwán

RECIBIDO: 28-10-2009

ACEPTADO: 02-12-2009 